\title{
Experimentation of Proactive Computing in Context Aware Systems: Case Study of Human-Computer Interactions in e-Learning Environment
}

\author{
Shirnin Denis, Reis Sandro, Zampunieris Denis \\ Computer Science and Communications Research Unit, \\ University of Luxembourg, \\ 6, rue Richard Coudenhove-Kalergi, L-1359, Luxembourg. \\ Email: \{denis.shirnin, sandro.reis, denis.zampunieris\}@uni.lu
}

\begin{abstract}
In this paper we show the implementation of the concept of Proactivity applied as the core mechanism in our Proactive Context Aware System (PCAS), which is capable to detect and extract the related events of interest from the user's contextual situation and to provide the appropriate goal-oriented actions to this event with the objective to help or assist the user or the group of users. We have chosen the academic environment as the ongoing contextual setting for our system. From this perspective, we designed Proactive Scenarios for an automatic and enhanced management of the online learning and teaching activities on Moodle ${ }^{\mathrm{TM}}$ for both student and teacher users. Due to the diversity of the potential contexts and situations arising from the user's activity, we developed two kinds of Proactive Scenarios. The first type or Meta Scenarios are responsible for capturing the changes in the outward context. The second type or Target Scenarios are triggered off by Meta Scenarios and aim to undertake the appropriate actions in response to the conditions of the user's contextual situation. In order to test and validate the capability of our software as well as to analyse its context related outcomes we have performed empirical studies. The experiments consisted in creating two groups of students, on the one hand the study group, which used the Moodle ${ }^{\mathrm{TM}}$ platform enhanced by PCAS, and on the other hand the control group, which used the standard version of Moodle ${ }^{\mathrm{TM}}$. The subsequent data analysis showed significant differences in specific related results such as notable advantage of the study group outcomes in the category of passing the final exam, where the study group has performed by 11 percentage points better than the control group.
\end{abstract}

Index Terms-Proactive System, Proactive Computing, Situational Awareness, Context Aware System, Context Situation Management, Cognitive Scenarios.

\section{INTRODUCTION}

The notion of Proactivity and Proactive Computing has been defined by Tennenhouse some time ago, however this branch in the research field of computer science is still considered as young [1], [2]. Throughout the study of its concept development, significant advantages of Proactive Systems in various domains of computer science have been highlighted [3], [4], [5]. One of such benefits that we show in the present paper is to be found in the field of Context Aware Systems. By its definition Proactive System has the capability to be aware of the surrounding context and to be able to act according to the needs of such context. In our opinion, the concept of Proactivity could significantly extend the research field that deals with Context Aware Systems by creating a link between its solid theoretical and empirical background and the new perspectives of not yet fully developed concepts, which are meant to bring the research in computer science further. Thus, in order to take this new concept into action we have chosen the academic environment as the most suitable and applicable in terms of implementation and subsequent performance testing. Concurrently, in such environment our Proactive Context Aware System (PCAS) allows us to detect the actual results, which are reflected through the students' e-learning activity.

\section{Proactive computing}

Being one of the promising fields in the computer science research, Proactivity implies mechanisms which in our opinion could enrich the potential of Context Aware Systems (CAS). The core mechanism of any CAS lies in the idea that implies the existence of three main elements Agent, Evolving situation and Situation analysis. In parallel, it includes the knowledge about the relationships between the various elements of an evolving situation. Such systems are able to provide adapted services for an agent through the situation analysis of gathered data from the evolving context [6], [7], [8]. On the other hand, the notion of Proactive Systems (PAS), as it was defined by Tennenhouse, takes the main approach of CAS further in its development [1], [2]. The main distinction between two types of systems could be considered as the ability of Proactive System to interact with the world around it, not only by providing the adaptive services, but also by bringing with it the aspect of proactivity, meaning that PAS is capable to perform appropriate actions on its own initiative depending on the evolving situation [4]. The second significant distinction is the type of sensors used by the system. As well as in the case of any CAS, Proactive System may use the implemented physical sensors to detect the changes in a surrounding context [9]. Besides these functions, PAS is able to use the situation development in itself as its input data, that is to use the preprogrammed Proactive Scenarios and to correlate them to the particular contextual situation, or an event [10]. In terms of 
proactive data analysis, a real life event as well as its absence in any giving context, are considered as equal. Thus, a nonevent of an outward context is treated by Proactive System as an event with its subsequent contextual outcomes. Various potential and actual contextual scenarios are used by Proactive System in order to build the appropriate target actions. This definition lets us make the third significant distinction between two types of systems. With the ability to use the real life scenarios of an evolving context as its sensors, PAS is capable to project its target actions with respect to the development of the future potential changes of a situation. Therefore, the concept of proactivity, in our opinion may significantly enrich the capabilities of CAS by providing its unique ability of putting the proactive aspect into action. This direction opens the doors for new potentials that could be applied in the research areas of Context Aware Systems and Ubiquitous Computing [11].

\section{Proactive CONTEXT AWARE System (PCAS)}

The initial concept of proactivity has encouraged researchers in computer science to support the approach further in its development. Thus, the idea of proactivity has emerged in the field of Learning Management Systems (LMS). By its definition, LMS are characterised as limited tools with its reactive and user-action oriented approach, which means the system does not act on its own initiative but waits for the user's commands in order to proceed further. On the other hand, integration of the concept of proactivity into the LMS provides an additional ability to act semi-autonomously or without explicit instructions from the user [12], [13]. In addition to the early mentioned advantages of PCAS implementation into the academic environment, some of the LMS platforms such as Moodle $^{\mathrm{TM}}$ have the ability to be integrated together with other software solutions. This point, represents for us another supportive argument in the decision making. By taking this point into consideration, we had in mind the objective, to create an enhanced e-learning environment by providing the basic Moodle's ${ }^{\mathrm{TM}}$ functions with the proactive type of behaviour, which allows to initiate intuitive and semi-autonomous actions dedicated to monitor the user's activity [14].

A better way to define the concept idea of the notion Context Aware is by fragmentation of the model in its individual parts. Thus, in our study we understand under the notion of Context the totality of different factors, types of users' online activities, diversity of academic tasks, types of management and organisation related problems, which constitute and form the comprehension of a setting that is subjected by our Proactive System in order to determine and to launch the appropriate actions. The notion Aware emerges from the software capabilities to identify the target contextual situations by referencing to the knowledge bank of the Proactive System database. In combination two sets of notions build the fundamental concept of our system, that is to identify and understand the surrounding context, analyse the situation with respect to the future potential development of a situation and to launch the appropriate actions. Such context aware features of PCAS are based on the variety of different Proactive Scenarios and rules, which reflect the actuality of a surrounding situation.

\section{A. PCAS integration and its prototype on Moodle ${ }^{T M}$}

Proactive System is represented by its integration into the currently used learning management platform Moodle ${ }^{\mathrm{TM}}$, which allows us to concentrate entirely on the development of PCAS by using already existing LMS as our framework.

In general representation of principal elements of the system, several basics could be highlighted. The main constituent that initiates the accurate functioning of proactive behaviours is the dynamic Rules Engine. This element represents the core of the system where all Proactive Scenarios and rules are analysed and processed [12], [13]. Another element of the system is a rule itself. In its variety and in the specific sets the rules represent contextually defined Proactive Scenarios (see section III-B). These elements provide our system with the feature of Proactivity and constitute the perception centre, which is responsible for the accurate detection of an event of interest and the intuitive subsequent initiation of the appropriate actions. In other words the system takes the proactive user-oriented approach, which consists of intuitive user-system interactions, helping, guiding, or assisting the user in his or her online learning activities. In Figure 1 we show the implementation of PCAS components into the Moodle $\mathrm{T}^{\mathrm{TM}}$ platform. Thus, we have the Rules Engine, which acts as it was defined in [13]. During its iteration period PAS executes the rules and provides us with the connection between the system and Moodle ${ }^{\mathrm{TM}}$, represented by a database wrapper. It allows the system to check the database for any relevant changes. We store the rules and some parameters needed for the running process in the Proactive System database in order to enable the system to restart in case of a problem. PCAS has three different types of parameters (F) - Time frequency of its activation periods; $(\mathrm{N})$ - The (maximum) number of rules it runs in an activation period, and (P) - The (minimum) time Proactive System pauses between two activation periods, which ensures that our system doesn't interfere with the LMS main process [10].

The last element that provides the visual features of the system is the graphical user interface which is displayed inside of the Moodle's ${ }^{\mathrm{TM}}$ webpage (see Figure 2). We have the main user interface dedicated to the students and the teachers, and the secondary user interface, which is used by the system administrator (currently under development).

\section{B. Proactive Scenarios}

If the Rules Engine is the main core of PCAS, alike Proactive Scenarios represent the second main element of the system. The schema of all potential situations is built on various scenarios issued from the real life context together with the abstraction and manipulation of its elements [10]. Each Proactive Scenario represents a set of rules where each rule is responsible only for a particular, single action. The complexity of a Scenario depends on the number of rules it encompasses. Proactive Scenarios are dedicated to accurately 


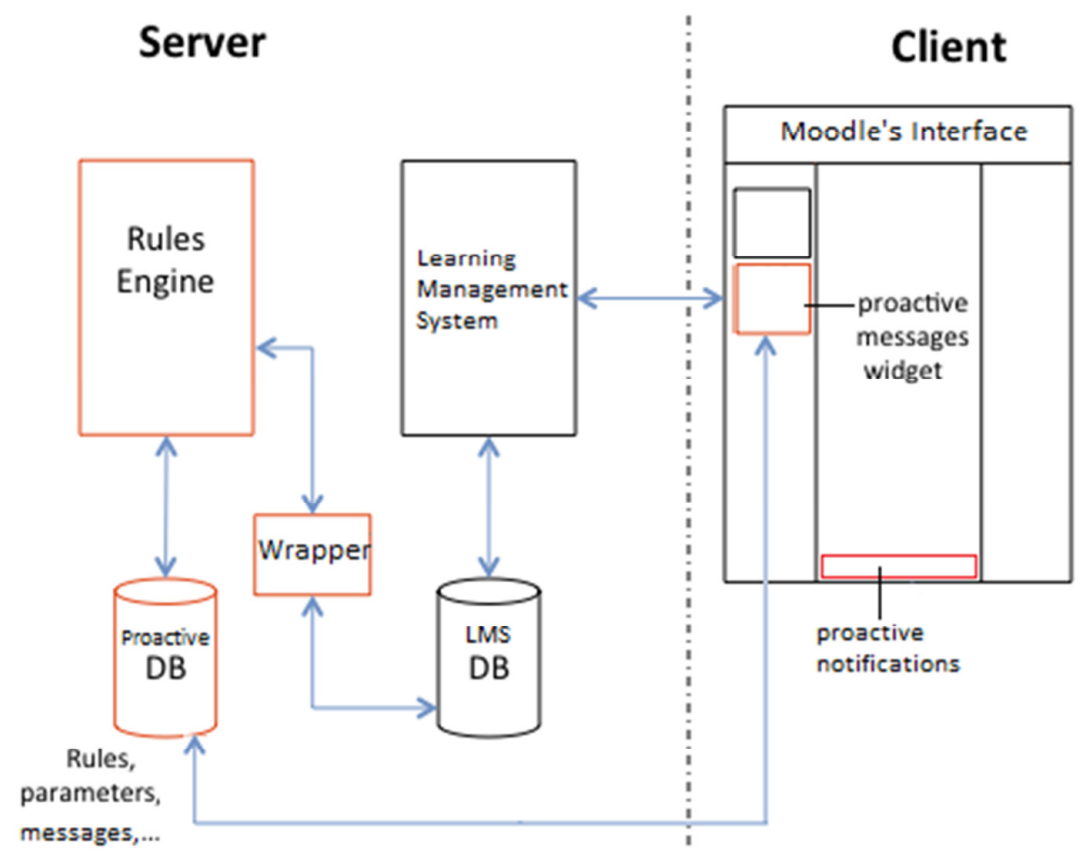

Fig. 1. System architecture

respond to any detected event of interest. Thus, Scenarios may vary in its features, essence, and complexity and therefore could be applied in various areas of a contextual situation.

For our Proactive System we have defined two types of Scenarios: type \#1 is the Meta Scenario and type \#2 is the Target Scenario. The name of Meta Scenario derives from the idea of meta-concept where one Scenario works for another, meaning that each Meta Scenario represents the higher level of a function, comparing to a Target Scenario. As being stated above, a set of specific rules constitute an individual and distinctive Proactive Scenario. Each Scenario has its own objective. In global view, it is either (1) to detect and extract an event from the context, or (2) to provide the appropriate services for each event, where such response-actions are needed. Therefore, Meta Scenarios are the Proactive Scenarios which are subjected to detect and extract an event from a context through the continuous analysis of the constantly changing outward environment. Tens or hundreds Scenarios of type \#1 could be launched in a group in order to be running simultaneously in various contextual situations. Thus, each Scenario will focus only on the detection of a particular and predefined outward condition for which it was programmed. It is vital to precise, that Meta Scenarios do not provide any response-actions to the detected event. This job is attributed to the Target Scenarios, which in their turn are launched by Meta Scenarios upon the detection of an event that needs to be treated through the set of the specific actions.

1) Type \#1 Meta Scenarios: Meta Scenario represents the perception centre of the system. It provides the system with the function of proactivity, which serves to detect and capture an event of interest. In order for PCAS to be aware about the contextual situation of a user it has first and foremost to be aware about the current state of the Moodle's ${ }^{\mathrm{TM}}$ database. In this optic, we turn to the tactic of the comparative analysis of data issued from the database. More specifically, in the frame of a time line, we compare the data and detect any changes, which constitute the abstraction of a context where the user's actions are involved. Thus, for each type of the data changes on the Moodle's ${ }^{\mathrm{TM}}$ database we have different Meta Scenarios, which by their definition run continuously in order to be constantly aware about any new data. All data changes represent an event of a real life situation. The main functionality of this type of Scenario is to be a context aware element of PCAS, which has to run continuously. After having detected the difference between the data, a new contextual situation for which it was programmed, the Meta Scenario launches the appropriate Target Scenarios for them to perform the specific actions in the real world. This process lets the Meta Scenario delegate the specific job to the appropriate Target Scenario. This type of actions defines the main character of Meta Scenario's functions, that is to provide the possibility of reciprocal interactions between the Proactive Context Aware System and the real world. The scope of its actions can be characterised as inward related because of its internal database analysis. However, the goal of its functions is fully oriented to the outer user's environment.

2) Type \#2 Target Scenarios: If Meta Scenario is characterised as inward related, the Target Scenario represents the outward actions of the system. The goal of the Target Scenario is to provide the appropriate response-actions for an event detected by a Meta Scenario. Type \#2 takes care of a unique target event by applying the predefined actions with an objective to provide the specific service type. In our case such services are Notifications, Reminders, Problem pre- 


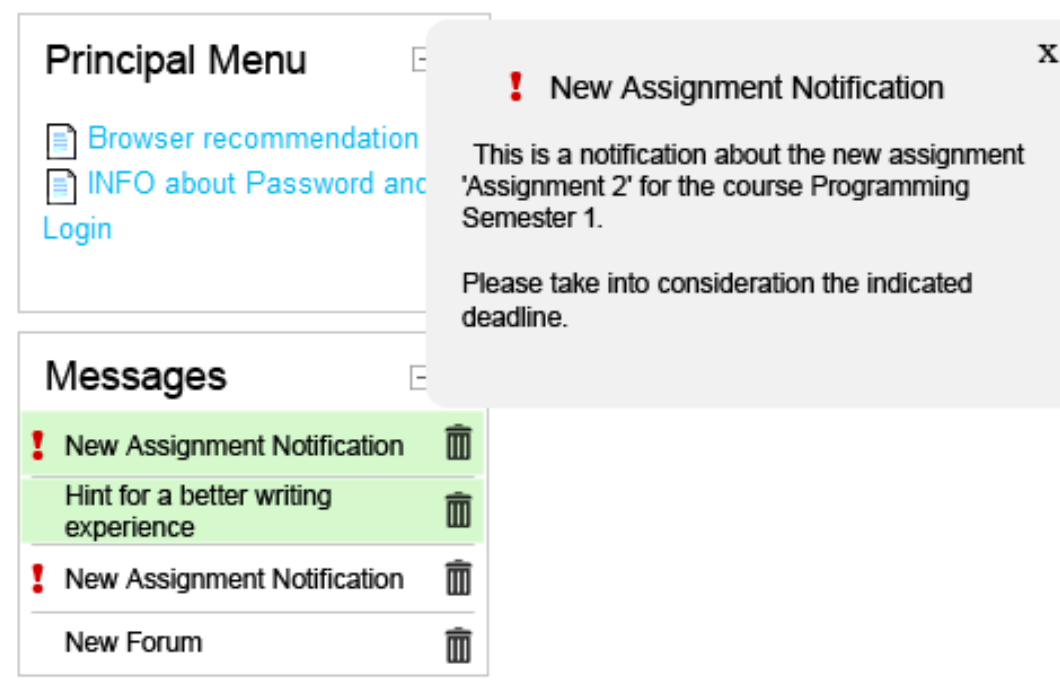

Fig. 2. PCAS target users' interface

vention, User guiding, etc. In a metaphorical perspective, the Meta Scenarios represent the brain process whereas the Target Scenarios are the hands of the system. The core distinction between two types of Proactive Scenarios is the condition of a Target Scenario to become dismissed as soon as it performs its unique job. The rules, which constitute the Scenario die after its execution. Such an approach lets us optimise our system in terms of memory usage.

Target Scenarios have as well their own areas of application. Thus, taking in consideration that any Target Scenario is characterised by its outward direction, the variety and type of its actions depend on a contextual situation and the relations between the elements of its environment. In our case, we define three different orientations: the system administrator environment, e-teacher environment, and e-student environment.

While creating each new Scenario we try to maximise the awareness of PCAS and its actions about the covered facets of human behaviour by studying the cognitive aspects of the user's intentions, objectives, and actions.

\section{Use of joined approaches}

In our project we use the expertise of two different domains Cognitive science and Computer science as we consider this integration beneficial. Throughout the research activity we have found that both fields have an interrelated and complementary effect.

While creating any Proactive Scenario we have to analyse the user's behaviour in various potential situations and to define its cognitive aspects. In order to perform this task, we apply the multitude of theories and approaches, which deal with the cognitive side of human behaviour. In our opinion, this type of interdisciplinary research allows us to build the accurately grounded scientific evidence for the further development of the concept definition of Proactivity and Proactive Systems.

The following brief description of theories and approaches focuses on the list of concepts used in the creation and conception of the Proactive Scenarios. It highlights the main orientations that we consider currently as the most relevant and important in terms of scientific rationalisation of new ideas by the cognitive science expertise.

Cognitive Approach and Behavioural Science allow us to study the aspects of user's behaviour and its foundations, which in its turn helps us to build the schema of possible users' actions in specific situations and to implement these aspects accordingly into Proactive Scenarios [15], [16].

Theory of Socially Shared Cognition is used in combination with the theory of Computer-user Interactions, which let us study the aspects of the computer-mediated interactions. By investigating the examples from social interactions we try to build the equivalent and applicable output related to the human-computer interactions [17], [18].

Activity Theory. Basing on the Higher Mental Functions Theory of Vygotsky we try to build the abstraction model of the cognitive functions of a user, which will help us to find and understand his or her potential behavioural patterns in various contextual situations [19], [20].

Theory of User's Identity is used in combination with previous theory where we try to build the picture of an average user and to identify its behavioural patterns [21], [22].

a) Example of theoretical implementation: Basing on the theoretical background highlighted above, we present different examples of PCAS behaviour in various situations such as the detection of an event of interest and interaction with the user by means of our messaging system. As we mentioned earlier, the extension of basic Moodle's ${ }^{\mathrm{TM}}$ functions has been amplified by proactive functions, which are mostly based on the cognitive patterns that reflect user's online activity and his or her e-learning contextual situation. In Table I we provide an example of some Scenarios that are currently used in our PCAS. The table specifies the type of a message sent to the user as well as its definition. Thus, by taking the message type Specific Assignment Hint, which has been sent for the 
TABLE I

SCENARIOS' SPECIFICATIONS

\begin{tabular}{|ll|}
\hline \multicolumn{1}{|c|}{ Message Type } & \multicolumn{1}{c|}{ Message definition } \\
\hline New event notification & System notifies the students about the new assignment. \\
\hline New event notification for teachers & System notifies the teacher that notification message has been sent to all students. \\
\hline Submission notification & System notifies the teacher about the level of $50 \%$ of the assignment submissions. \\
\hline Writing tasks suggestions & $\begin{array}{l}\text { System provides the students with the information/hints on how to better perform the writing tasks. The } \\
\text { message contains a list of external online sources that are specialised on the explanations of planning, } \\
\text { structuring, and writing techniques. }\end{array}$ \\
\hline Specific assignment hint & System sends to the students the hints provided by a teacher for a particular assignment. \\
\hline Reminder of an assignment submission & $\begin{array}{l}\text { System reminds the students about the deadline of an assignment if they have not yet submitted their } \\
\text { works by the specified time. }\end{array}$ \\
\hline Reminder of the upcoming event & System reminds the students and the teachers about the upcoming event detected on the calendar. \\
\hline Summary of the upcoming events & System reminds the students and the teachers about the upcoming events of the next week. \\
\hline Potential problem & System informs the teacher about the potential problem concerning his/her assignment. \\
\hline Problem detection & System informs the teacher about the problem concerning his/her assignment. \\
\hline
\end{tabular}

assignment $L$ interpreter in $C$ and comparing it with the data on Table II, we may notice the significant difference between the submission results of two groups, $22.2 \%$ for the control group and $77.8 \%$ for the study group. This example represents the outcome of the proactive behaviour of PCAS oriented to guide a user in his or her e-learning activity.

\section{EXPERIMENTATION DEFINITIONS}

It is important to explore and build the theoretical background of a study, and it is equally important to provide empirical evidence of a study by subjecting its constituent elements to testing and experimentations. Our survey aims to test and verify the claims and positions of all the approaches, theories and concepts used in the project as well as the performance and stability of the Proactive Context Aware System.

\section{A. Experiments objectives and its focal points}

The conceptual problems and their various study elements, which have to be scrutinised and expounded, constitute the main goal of the experiments.

We have distinguished two global types of problem sets that enclose the aspects from two different study fields, computer science and cognitive science. The former aims to examine the issues related to the software and general system performance, the latter targets the learning related issues together with a general user's perception of various system functions.

Software related fields. We differentiate two types of software related fields, which are covered in our survey, and in automatic statistical data gathering. The first field comprises the issues of general character such as functions variety, its quality, performance of e-learning resources, and ease of software usability. The second field deals with the visual aspect of the software that is the user interface, its elements, position, colour, and effectiveness of functions. We extend the software related part of the survey by various types of automatic statistics, which aim to highlight the users' perception of new functions, the willingness to use it, the level of user's productivity while working online, and their learning interests and habits related to the use of specific functions of Learning Management System. We gather as well some technical statistics, which mostly reflect the system performance.

Learning related fields. The second type of problem set deals with the issues related to the process of e-learning, and e-teaching. The survey aims to gather information, which encompasses such cognitive directions as the practice of elearning activity, cooperative and collaborative learning, learning competences, performance, and motivation. The data, which reflects the characteristics of these types of activities, will allow us to build more authentic cognitive-oriented Scenarios and rules, as well as to extend the capabilities of the Proactive Context Aware System for better intuitiveness and sensitivity regarding the users' goals in the variety of specific contexts.

At the present, both layers of survey constitute the entirety of issues that in our opinion have to be studied and analysed in order to perform the following concept improvement and overall system optimisation.

\section{B. Experiments structure and methodology}

In the previous sections we covered the general highlights of the experiments where we have specified and described two problem sets. Thus, in order to uncover the details of the experiments, we have to define first all the necessary steps, tools, and methods that have to be undertaken.

The methodology of our experiments consists of several types of activity. The primary steps involve the process of plan creation where we define all basic and compulsory proceedings. Thus, we decided to carry the experiments during 
TABLE II

SUBMISSION LEVEL

\begin{tabular}{|c|c|c|c|c|c|c|c|c|c|c|c|}
\hline \multirow[b]{2}{*}{ course } & \multirow[b]{2}{*}{ assignment } & \multirow{2}{*}{\multicolumn{3}{|c|}{$\begin{array}{l}\text { \# enrolled students } \\
\text { total control study }\end{array}$}} & \multicolumn{6}{|c|}{ \# submitted } & \multirow[b]{2}{*}{ diff } \\
\hline & & & & & total & $\%$ & control & $\%$ & study & $\%$ & \\
\hline Algorithmics 2 & Toll System (Interface & 18 & 9 & 9 & 8 & $44.4 \%$ & 4 & $44.4 \%$ & 4 & $44.4 \%$ & \\
\hline Algorithmics 2 & Toll System & 18 & 9 & 9 & 9 & $50.0 \%$ & 4 & $44.4 \%$ & 5 & $55.6 \%$ & \\
\hline Algorithmics 2 & Heuristic for & 18 & 9 & 9 & 10 & $55.6 \%$ & 4 & $44.4 \%$ & 6 & $66.7 \%$ & \\
\hline Algorithmics 2 & $\mathrm{~L}$ interpreter in $\mathrm{C}$ & 18 & 9 & 9 & 9 & $50.0 \%$ & 2 & $22.2 \%$ & 7 & $77.8 \%$ & \\
\hline \multicolumn{2}{|c|}{ Algorithmics 2} & 18 & 9 & 9 & & $50.0 \%$ & & $38.9 \%$ & & $61.1 \%$ & $22.2 \%$ \\
\hline Probabilities & Exercises 2 & 41 & 20 & 21 & 22 & $53.7 \%$ & 9 & $45.0 \%$ & 13 & $61.9 \%$ & \\
\hline Probabilities & Exercises 3 & 41 & 20 & 21 & 30 & $73.2 \%$ & 12 & $60.0 \%$ & 18 & $85.7 \%$ & \\
\hline Probabilities & Exercises 4 & 41 & 20 & 21 & 8 & $19.5 \%$ & 3 & $15.0 \%$ & 5 & $23.8 \%$ & \\
\hline \multicolumn{2}{|c|}{ Probabilities } & 41 & 20 & 21 & & $48.8 \%$ & & $40.0 \%$ & & $57.1 \%$ & $17.1 \%$ \\
\hline \multicolumn{2}{|c|}{ TOTALS } & 59 & 29 & 30 & & $49.5 \%$ & & $39.4 \%$ & & $59.4 \%$ & $20.0 \%$ \\
\hline \multicolumn{2}{|c|}{ Probabilities (without exercise 4) } & & & & & $63.4 \%$ & & $50.0 \%$ & & $77.5 \%$ & $27.5 \%$ \\
\hline \multicolumn{2}{|c|}{ TOTALS (without exercise 4) } & & & & & $54.5 \%$ & & $43.4 \%$ & & $65.3 \%$ & $21.9 \%$ \\
\hline
\end{tabular}

one semester where we employ two different courses of a bachelor level. During this period the students have altogether 7 assignments that our system has to treat with its proactive functions. We divided the students into two groups, a study group and a control group. The study group or proactive group that has to be subjected to our experimentations, represents the key group. The process of group creation consisted of students' quality categorisation where we established the list of students regarding their previous grades. The second step consisted of balancing two groups with relation to our list meaning that each group had to include an equal number of students both with good grades as well as with bad ones. The participants of the study group have been subjected to use the integrated proactive functions of Moodle ${ }^{\mathrm{TM}}$. The control group used the standard version of Moodle ${ }^{\mathrm{TM}}$ with the default functions. Thus, at the end of the experiments we are able to confront and to test the results and any related data derived from the initial group. Therefore, the interest of the survey focuses on the development of appropriate measurement tools, which have to be subsequently applied to studying and analysing the aspects of the study group activity. In order to allocate all steps proportionally throughout the whole semester, we have defined a schema with different phases, where each phase consisted of specific steps and actions. Consequently, we have three focal phases; the first includes the preliminary study where we have to create two groups of students, one proactive profile for each course, and a first questionnaire that highlights the general user's perception of Moodle's ${ }^{\mathrm{TM}}$ functions. The second phase of our experiment mainly consists of various statistical data collection, which highlights both the system optimisation issues and users' e-learning activity aspects. At this point we create the second questionnaire that aims to build the schema of users' views and opinions regarding the new proactive functions of Moodle ${ }^{\mathrm{TM}}$. In the survey we use a multiple-choice, open-ended type of questionnaire where we give the possibility to the user either to choose the predefined answers or to write his or her own. The third phase includes the consequent questionnaire analysis, statistical data analysis, as well as the ultimate comparison of students' academic results in both groups.

\section{EXPERIMENTATION RESULTS}

Basing on previously stated goals of our experiments and subsequent data analysis we have defined three types of outcomes, that is, (a) learning related, (b) user-computer interaction related, and additionally (c) system related. In its totality the three layers of outcomes provide us a global picture of the results.

\section{A. Learning related}

As we mentioned earlier, in section III-C each created Proactive Scenario aims to reflect the cognitive aspect of human behaviour related to the specific user's learning activity, therefore each message that our system sends has its own predefined objective. Some of the Scenarios such as New event notification or Reminder of an assignment submission are meant to stimulate the students' learning efficacy in their quest of assignment accomplishment and its final submission. The statistics, which highlight the results of this activity are shown in Table II. During the experiments we have gathered data only from two courses Algorithmics 2 and Probabilities, which have 7 assignments in total. We have 18 students for the course Algorithmics 2 where 9 students belong to the control group and another 9 to the study group. For the course Probabilities we have 41 students in total where 20 constitute the control group and 21 constitute the study group. First we take a look at the results, which represent the percent of submissions by a course. In Algorithmics 2, the 50\% of 
TABLE III

STUDENTS' FINAL GRADES

\begin{tabular}{|c|ccc|cc|cc|cc|c|}
\hline & \multicolumn{7}{|c|}{ \# enrolled students } & \multicolumn{7}{|c|}{ \# passing students } & diff \\
\cline { 2 - 13 } course & total & control & study & total & $\%$ & control & $\%$ & study & $\%$ & \\
\hline Algorithmics 2 & 18 & 9 & 9 & 3 & $16.7 \%$ & 0 & $0.0 \%$ & 3 & $33.3 \%$ \\
\hline Probabilities & 41 & 20 & 21 & 29 & $70.7 \%$ & 14 & $70.0 \%$ & 15 & $71.4 \%$ & \\
\hline \hline TOTALS & 59 & 29 & 30 & 32 & $54.2 \%$ & 14 & $48.3 \%$ & 18 & $60.0 \%$ & $11.7 \%$ \\
\hline
\end{tabular}

total submissions include $38.9 \%$ for the control group, and $61.1 \%$ for the study group. Thus we notice the advantage of the study group, which is represented by 22.2 percentage points. For the course Probabilities we have a similar picture, which shows the advantage of the study group results over the control group results by 17.1 percentage points. In total for both courses we have $39.4 \%$ of submissions for the control group and $59.4 \%$ for the study group with an advantage of 20.0 percentage points for the second group. However, if we take a look on the data, which represents the submission values for the course Probabilities, assignment Exercices 4 we may notice significantly low number of submissions for both groups, 3 and 5 respectively. Because of this incomplete picture, which could result from the assignment submissions off Moodle ${ }^{\mathrm{TM}}$ platform, we decided to analyse the difference without the assignment Exercices 4. In this optic, we have $50.0 \%$ of submissions for the control group and $77.5 \%$ for the study group or in total $43.4 \%$ and $65.3 \%$ respectively, where 21.9 percentage points is the advantage value of the study group. The data, which represents the results by assignment shows as well a considerable advantage of the study group for almost each assignment of the two courses except the first assignment of the course Algorithmics 2 where the results have the same value.

In Table II we saw the data, which displays only one type of a learning activity, the assignment submission. In order to gain more objectiveness in our results we decided to use the global data of the whole academic semester, that is to analyse the students' final grades for two mentioned courses. In this perspective the grades represent the global scale character of our data analysis. As in the previous example, the results are divided by course and by type of group (see Table III). Thus, we have for the course of Probabilities 70\% of students of the control group who passed the final exam and $71 \%$ of passed students of the study group. For the course of Algorithmics 2 the result is $0 \%$ of passed students for the control group and $33 \%$ of passed students for the study group. By comparing the results, in total for each group we get $48 \%$ of control group students who passed the final exam and $60 \%$ of passed students for study group. Once more, we may notice a clear advantage of the study group over the control group in the category of the students' final grades.

These outcomes reflect the significant advantage of the study group results in another type of statistics, which highlight the total time spent online on Moodle ${ }^{\mathrm{TM}}$ platform during one semester for each group. Thus, we have 225.3 hours of spent time for the control group and 469.5 hours for the study group.

The results of three types of statistics mentioned above, representing the learning related part of the experiments, point to the important impact of the extended Moodle's ${ }^{\mathrm{TM}}$ functions, which are provided by the proactive behaviour of PCAS. This positive outcome of Proactive System reflected by the success level of students who used the LMS with enhanced functions confirm the same conclusions already suggested in [23] by a similar study.

\section{B. User-computer interaction related}

Both, the perception of graphical user interface as well as the interaction with it play an important role in the system's optimisation process. Therefore, the second layer of our statistics consists of data gathered in relation to this issue.

In order to provide the interaction related aspect of PCAS functioning we have implemented the graphical user interface, which is built as an additional management block on Moodle's ${ }^{\mathrm{TM}}$ webpage. It allows the user to interact with PCAS by simple commands such as open or delete a message, open and see the list of messages, or just see the headlines of unread messages. For statistical purposes we gathered the data, which highlights the details of user interactions with our implemented proactive block inside of the Moodle's ${ }^{\mathrm{TM}}$ webpage. The data shows that during one semester there have been 251 explicit interactions with the proactive block, including actions of opening a message and deleting it either prior to viewing or after it. In addition we have 224 messages that show no explicit interactions, meaning the user neither deleted or opened a message. However it may indicate that the user has read at least the heading of a message.

Together with the statistics, we have collected supplementary data issued from the questionnaires, which highlights the users' perception and opinions related to the visual and interactive aspects of the PCAS user interface. Due to the low rate of received answers we have decided not to use the questionnaire data for any quantitative analysis but rather to take it into consideration for qualitative analysis during the next optimisation process.

\section{System related}

As we mentioned earlier in section III-A Proactive Context Aware System has an iteration period during which the rules are executed. The statistics described in the present section are 
related to the system performance where it shows the data of various technical issues.

Thus, the data shows for the running time of one semester Proactive System performed 772050 iterations where it executed 6151617 Meta Scenarios and 1326 Target Scenarios. In total, 475 messages have been sent by PCAS. In average one iteration took 20 milliseconds during which between 4 and 15 rules have been executed. It includes as well the access of PCAS into the Moodle's ${ }^{\mathrm{TM}}$ database.

\section{CONCLUSIONS}

In the present paper we have shown the results, which highlight the experiments of Proactive Computing in Context Aware Systems. We implement the concept of proactivity in the frame of the theories, which define the notion of Situation Awareness. The experiments, which took place during one academic semester have shown a significant difference in the results of both groups. Thus, we have found an advantage of study group results in several categories such as intensity of students' actions related to the learning activity as well as their level of accomplishment represented by the final grades. The data, which has been presented in section $\mathrm{V}$, shows the impact of proactive behaviour integrated as the core mechanism for the context aware capabilities of a system. The fusion of both, computer science expertise together with the cognitive science, has contributed to the scientific evidence of the study as well as to the conceptualisation of the project and its output. The obtained experiment's results have pushed us to extend the phase of experiments by preparing and organising a second set of observations where we plan to enhance the capability of PCAS by implementing new collaborative features, and to expand the capacity of its perception centre by using the supplementary sensors and detectors.

We believe that the potential of proactivity, if integrated into the framework of Context Aware Systems, may provide significant contributions to this field and open doors for new research prospects.

\section{REFERENCES}

[1] D. Tennenhouse, "Proactive computing," Communications of the ACM, vol. 43, no. 5, pp. 43-50, May 2000.

[2] R. Want, T. Pering, and D. Tennenhouse, "Comparing autonomic and proactive computing," IBM Systems Journal, vol. 42, no. 1, pp. 129-135, 2003.

[3] Y. Engel and O. Etzion, "Towards proactive event-driven computing," in Proceedings of the 5th ACM international conference on Distributed event-based system, ser. DEBS '11. New York, NY, USA: ACM, 2011, pp. 125-136.

[4] G. Vlahakis and D. Apostolou, "Proactivity in service based applications," Informatics (PCI), 2012 16th Panhellenic Conference on, pp. 62-67, 2012.
[5] O. Kwon, K. Yoo, and E. Suh, "Ubidss: a proactive intelligent decision support system as an expert system deploying ubiquitous computing technologies," Expert Systems with Applications, vol. 28, no. 1, pp. 149161, 12005.

[6] C. Matheus, M. Kokar, and K. Baclawski, "A Core Ontology for Situation Awareness," in 6th International Conference on Information Fusion, 2003

[7] Y. Uhm, Z. Hwang, M. Lee, Y. Kim, G. Kim, and S. Park, "A ContextAware Dynamic Service-Oriented System for Reconstructing Adaptive Services by the Management of Rule and Ontology," 2007 International Conference on Convergence Information Technology (ICCIT 2007), pp. 83-88, Nov. 2007.

[8] J. B. Filho and N. Agoulmine, "A Quality-Aware Approach for Resolving Context Conflicts in Context-Aware Systems," 2011 IFIP 9th International Conference on Embedded and Ubiquitous Computing, pp. 229-236, Oct. 2011.

[9] T. Timpka, "Proactive health computing," Artificial Intelligence in Medicine, vol. 23, no. 1, pp. 13-24, 82001.

[10] S. Reis, D. Shirnin, and D. Zampunieris, "Design of proactive scenarios and rules for enhanced e-learning," in CSEDU 2012 - Proceedings of the 4th International Conference on Computer Supported Education. Porto, Portugal: SciTePress, 2012, pp. 253-258.

[11] O. Kwon, "The potential roles of context-aware computing technology in optimization-based intelligent decision-making," Expert Systems with Applications, vol. 31, no. 3, pp. 629-642, 102006.

[12] D. Zampunieris, "Implementation of a Proactive Learning Management System," in E-Learn World Conference on E-Learning in Corporate, Government, Healthcare and Higher Education, Hawaii, 2006, pp. 3145-3151.

[13] D. Zampunieris, "Implementation of Efficient Proactive Computing Using Lazy Evaluation in a Learning Management System," International Journal of Web-Based Learning and Teaching Technologies, vol. 3, no. 1, pp. 103-109, 2008.

[14] S. M. Dias, S. Reis, and D. Zampunieris, "Personalized, Adaptive and Intelligent Support for Online Assignments Based on Proactive Computing," in 2012 IEEE 12th International Conference on Advanced Learning Technologies. Rome, Italy: IEEE, Jul. 2012, pp. 668-669.

[15] F. Gao, M. Li, and Y. Nakamori, "Critical systems thinking as a way to manage knowledge," Systems Research and Behavioral Science, vol. 20, no. 1, pp. 3-19, Nov. 2002

[16] B. Burnes, "Complexity theories and organizational change," International Journal of Management Reviews, vol. 7, no. 2, pp. 73-90, Jun. 2005.

[17] S. A. Siler, "Learning, Interactional, and Motivational Outcomes in Oneto-One Synchronous Computer-mediated versus Face-to-Face Tutoring," International Journal of Artificial Intelligence and Education, vol. 19, no. 1, 2009

[18] B. Wrede, S. Kopp, K. Rohlfing, M. Lohse, and C. Muhl, "Appropriate feedback in asymmetric interactions," Journal of Pragmatics, vol. 42, no. 9, pp. 2369-2384, 2010.

[19] L. S. Vygotsky, "The genesis of higher mental functions," in The concept of activity in Soviet psychology, J. V. Wertsch, Ed. Armonk, NY: Sharpe, 1981, pp. 144-188.

[20] B. A. Nardi, "Context and consciousness: Activity theory and humancomputer interactions," MIT Press, 1996.

[21] D. H. Zimmerman, "Identity, context and interaction," in Identities in Talk, C. Antaki and S. Widdicombe, Eds. London: Sage, 1998, pp. 87-106.

[22] M. Rowe, "The credibility of digital identity information on the social web: a user study," in Proceedings of the 4th workshop on Information credibility. ACM, 2010, pp. 35-42.

[23] S. Coronado and D. Zampunieris, "Continuous Proactivity in Learning Management Systems," in IEEE EDUCON 2010 Conference, 2010, pp. 199-204. 